\title{
Letter to the Editor: Classification of Pituitary Adenomas
}

\author{
T. Mindermann \\ Department of Neurosurgery, University Hospitals Basel, Basel, Switzerland
}

In their article, Sanno et al. [15] address an important issue concerning the classification of pituitary adenomas. Their proposed classification is based on the tumour's histological data. Yet, pituitary adenomas are treated by a number of specialists, not all of whom have histological data available. Therefore, I would like to present an alternative classification scheme which can be shared by endocrinologists, gynaecologists, ophthalmologists, neurosurgeons, pathologists, and researchers alike.

Advances in the understanding of an adenoma's hormone synthesis (genotype), hormone storage (immunophenotype), and hormone release into the blood stream (clinical phenotype) have lead to several parallel and sometimes confusing classifications of pituitary adenomas. The present confusion on what exactly is, e.g., a prolactinoma or a corticotroph cell adenoma (does it produce, store, or release ACTH?) has lead to the occasional use of such terms as "PRLoma", "GH-oma", "ACTH-oma", and "TSH-oma" [2, 16] or "somatotropinoma" and "corticotropinoma" [3]. Unfortunately these terms do not clarify whether in the case of "PRL-omas" the adenomatous cells produce, store, or release PRL or if the excessive release of PRL is caused by the stalk effect or raised intrasellar pressure which would mean that the adenomatous cells do not even derive from the mammotroph cell line. Currently, due to these difficulties and to overlap created by the various terminologies, tumours of the same cell line may be classified differently depending on whether one refers to their genotype, immunophenotype, or clinical phenotype. Vice versa, tumours deriving from different cell lines may incorrectly be classified as identical tumours. In an attempt to overcome the difficulties in making a correct diagnosis based on clinical data, some authors have developed regression equations in order to extract most information from the clinical data [5]. The introduction of mathematical tools to improve the accuracy of the clinical diagnosis demonstrates the great difficulties encountered when trying to determine an adenoma's true nature in the absence of histological data. On the other hand, classifications based on a tumour's histological examination can often not be shared with clinicians who treat their patients before or without surgery.

Therefore, I would like to emphasize an unequivocal classification of the various adenoma types by crossreferencing an adenoma's cell line of origin, its immunoreactivity, and its clinical phenotype. At the same time, existing anatomical classifications should be used to complement this classification. The aim is to come to a diagnosis that can be shared by researchers, pathologists, and clinicians.

\section{Proposed Classification of Pituitary Adenomas}

The system is based on the work by Kovacs [8] and Landolt [9] whose contributions replaced the classical histological designation of pituitary adenomas as acidophilic, basophilic, and chromophobic with the more meaningful designation as adenomas of a certain anterior lobe cell line. It takes into account new findings that link somatotroph, mixed mammotroph and somatotroph, and mammosomatotroph adenomas to one basic cell line committed to the production of growth hormone [12].

The classification presented in Table 1 has been published elsewhere [18]. It takes into account an adenoma's direct and indirect (stalk effect, raised 


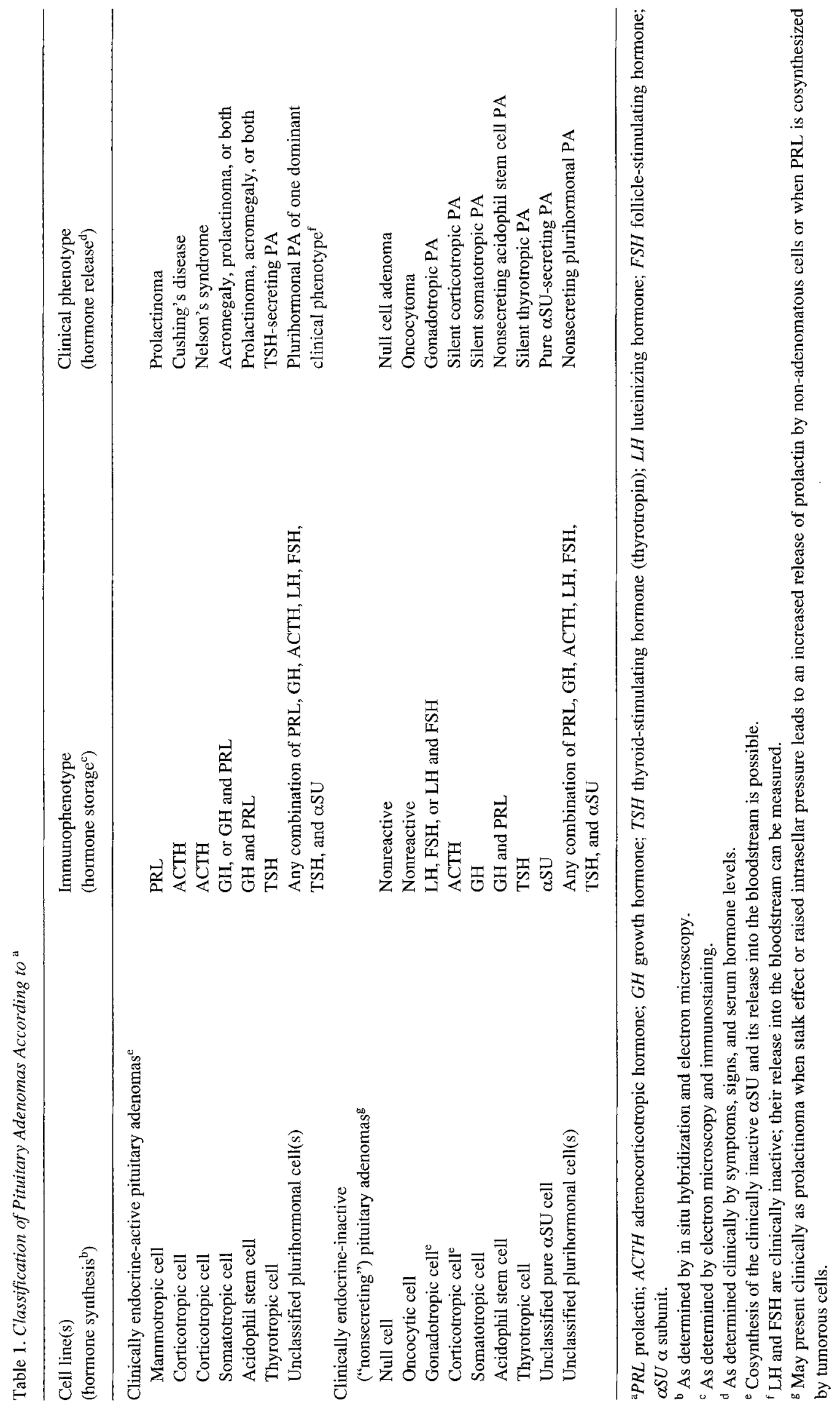


intrasellar pressure) endocrine activities. The direct endocrine activities are: hormone synthesis (genotype), hormone storage (immunophenotype), and hormone release (clinical phenotype). The indirect endocrine activities are: compression of non-adenomatous anterior lobe tissue resulting in partial pituitary insufficiency and excessive release of PRL by non-adenomatous cells caused by the stalk effect or raised intrasellar pressure.

The genotype is determined by molecular genetic techniques like in-situ hybridization [13] allowing one to identify both DNA and mRNA in adenomatous cells revealing their genetic fingerprint. It is the most accurate information obtainable concerning the cell line of origin and is mostly used in research.

The immunophenotype is determined by the tumour's immunostaining properties when adenomatous tissue is stained with antibodies to the various anterior lobe hormones or hormonal compounds such as the alpha-subunit. This technique reveals which hormone(s) or hormonal compound(s) are stored by tumorous cells. It is a less accurate way of determining an adenoma's cell line of origin since hormonal products may be released without the delay of tissue storage. Immunostaining for the various anterior lobe hormones should be part of today's routine diagnostic procedures.

The clinical phenotype is the least accurate information conceming the nature of a pituitary adenoma because: (a) hormone excess may be insufficient to cause any clinical symptoms or signs, (b) release of hormonal products may be clinically silent, (c) due to raised intrasellar pressure or the stalk effect prolactin may be released in sufficient amounts to cause amenorrhea and galactorrhea even though the tumour might not produce prolactin $[1,10,11]$, (d) the clinical phenotype may be determined by only one of multiple co-existing tumours of differing immunophenotypes [7], (e) tumours may change clinical and immunophenotype [14], and (f) in addition to hormone excess, tumours may cause partial pituitary insufficiency.

The proposed classification scheme keeps the doctor aware of the degree of certainty concerning the true nature of a pituitary adenoma. For obvious reasons, a classification of a patient's adenoma based on clinical data only leads to the least reliable diagnosis. One might grade the three groups represented in the three columns to which a pituitary adenoma may be designated as fair evidence (clinical phenotype), good evidence (immunophenotype), or best evidence (cell line) for the correct diagnosis.

The anatomical classification schemes developed by Hardy [4] and modified by Wilson [17] grade the tumour's extent of sellar destruction and its extrasellar extension. A newly developed classification by Knosp et al. [6] based on magnetic resonance imaging (MRI) grades an adenoma's invasion into the cavernous sinus space. At surgery, tumour extending beyond the intercarotid line in coronal MRI proved very likely to be associated with invasion [6]. These purely anatomical classifications should be used in addition since the data contained in these schemes have prognostic value and are helpful in choosing the best treatment.

The proposed classification in combination with the various grading schemes should facilitate interdisciplinary and interinstitutional communication on what tumour exactly clinicians, pathologists, different institutions and articles in scientific publications are referring to. It is designed to highten the awareness that there are several aspects characterizing a pituitary adenoma ranging from its cell line of origin to its immunophenotype and to its clinical presentation. A hightened awareness of such aspects may help in improving study designs and in the understanding of the tumour biology and the clinical course of the various adenoma types.

\section{References}

1. Anonymous (1987) Hyperprolactinaemia. When is a prolactinoma not a prolactinoma? Lancet 2: 1002-1004

2. Beck-Peccoz P (1993) Unusual presentation of a TSH-secreting pituitary adenoma. Acta Endocrinol 129: 283

3. Faglia G (1993) Epidemiology and pathogenesis of pituitary adenomas. Acta Endocrinol 129 [Suppl 1]: 1-5

4. Hardy J, Wigser SM (1965) Trans-sphenoidal surgery of pituitary fossa tumors with televised radiofluoroscopic control. J Neurosurg 23: 612-619

5. Herruzo-Cabrera I, Herruzo-Cabrera R, Errazquin-Saez de Tejada L, Garcia-Fernandez JL, Vidarte-Zabala M, Mayer F, Zaragoza-Rubira JR (1992) A multivariant study of pituitary adenoma, obtainment of two logistic regression equations as an auxiliary support in the diagnosis of these tumors. Neoplasma 39: $255-260$

6. Knosp E, Steiner E, Kitz K, Matula C (1993) Pituitary adenomas with invasion of the cavernous sinus space: a magnetic resonance imaging classification compared with surgical findings. Neurosurgery 33: 610-618

7. Kontogeorgos G, Scheithauer BW, Horvath E, Kovacs K, Lloyd RV, Smyth HS, Rologis D (1992) Double adenomas of the pituitary: a clinicopathological study of 11 tumors. Neurosurgery $31: 840-849$

8. Kovacs K, Horvath E (1986) Tumors of the pituitary gland. In: 
Hartmann WH, Sobin LH (eds) Atlas of tumor pathology. Armed Forces Institute of Pathology, Washington DC, pp $1-252$

9. Landolt AM (1975) Ultrastructure of human sella tumors. Correlation of clinical findings and morphology. Acta Neurochir (Wien) [Suppl] 22: 1-167

10. Lees PD (1990) Intrasellar pressure. Acta Neurochir (Wien) [Suppl] 47: 68-70

11. Lees PD, Pickard JD (1987) Hyperprolactinemia, intrasellar pituitary pressure, and the stalk compression syndrome. J Neurosurg 67: 192-196

12. Li J, Stefaneanu L, Kovacs K, Horvath E, Smyth HS (1993) Growth hormone $(\mathrm{GH})$ and prolactin (PRL) gene expression and immunoreactivity in GH- and PRL-producing human pituitary adenomas. Virchows Archiv A Pathol Anat 422: 193-201

13. Lloyd RV, Jin L, Chandler WF (1991) In situ hybridization in the study of pituitary tissues. Path Res Pract 187: 552-555

14. Mindermann T, Kovacs K, Wilson CB (1994) Changes in immunophenotype of recurrent pituitary adenomas. Neurosurgery 35 : $39-44$

15. Sanno N, Teramoto A, Osamura RY (1996) Clinical and cytofunctional classification of pituitary adenomas: proposal of a new classification. Acta Neurochir (Wien) 138; 1186-1192

16. Spada A, Vallar L, Faglia G (1994) Cellular alterations in pituitary tumors. Eur J Endocrinol 130: 43-52

17. Wilson CB (1983) Surgical management of endocrine-active pituitary adenomas. In: Walker MD (ed) Oncology of the nervous system. Martinus Nijhoff, Boston, pp 117-150

18. Wilson CB, Mindermann T (1997) Pituitary neoplasms. In: Holland JF, et al (eds) Cancer medicine, 4th Ed. Williams and Wilkins, Baltimore, pp 1539-1550

Correspondence: Dr. Thomas Mindermann, Department of Neurosurgery, University Hospitals Basel, CH-4031 Basel, Switzerland. 